Arquitectura +

ISSN: 2518-2943

www.revistas.uni.edu.ni/index.php?journ

al=arquitectura

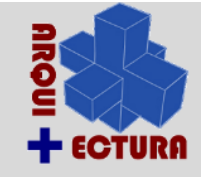

Vol. 1- Número 2 / Diciembre 2016

Gestión de riesgo con enfoque en ordenamiento territorial en el casco urbano del municipio de San Nicolás, departamento de Estelí/

Paizano, Martha Alicia/ marthapaizano@gmail.com

\title{
Artículos
}

\section{Gestión de riesgo con enfoque en ordenamiento territorial en el casco urbano del municipio de San Nicolás, departamento de Estelí}

\section{Risk management with a focus on land use in the urban area of the municipality of San Nicolás, department of Estelí \\ Paizano, Martha Alicia (marthapaizano@gmail.com)}

\begin{abstract}
The urban center of the municipality of San Nicolás, Estelí presents geological and climatic characteristics that propitiate the occurrence of multiple slope movements such as: translational landslides, rotational, complex; Also sinkings, lateral separations and falls of debris. These events put at risk the life of the settlers, due to the conditions of vulnerability with which they coexist in their environment. Due to the relevance of this threat, this study is focused on reducing the risks arising from slope movements, through the implementation of a risk management plan.

The results of the study of Risks to slope movements of González (2011) were taken in the same study area, whose main result was a risk map, which was a starting point for the realization of territorial zoning.

The risk management plan contains four development axes with a focus on disaster reduction: economic, social, institutional and capacity building, land management and the environment. It is in this last axis that integrates the territorial ordering in the management plan; It is proposed the zoning of the territory in function of the risk by movements of slopes, based on Decree 78-2002: Of Norms, Guidelines and Criteria for the Territorial Ordering.
\end{abstract}

\section{Keywords}

Threats, risk management, land use, vulnerability, Estelí

\section{Resumen}

El casco urbano del municipio de San Nicolás, Estelí presenta características geológicas y climáticas que propician la ocurrencia de movimientos múltiples de laderas como: deslizamientos translacionales, rotacionales, complejos; también hundimientos, separaciones laterales y caídas de detritos. Dichos eventos ponen en riesgo la vida de los pobladores, por las condiciones de vulnerabilidad con las que coexisten en su entorno. Debido a la relevancia que supone esta amenaza, este estudio está enfocado en la reducción de los riesgos provenientes de los movimientos de laderas, a través de la realización de un plan de gestión de riesgo.

Se tomaron los resultados del estudio de Riesgos ante movimientos de laderas de González (2011) en la misma área de estudio, cuyo principal resultado fue un mapa de riesgos, el cual fue un punto de partida para la realización de la zonificación territorial.

El plan de gestión de riesgos contiene cuatro ejes de desarrollo con enfoque a la reducción de desastres: económico, social, institucionalidad y desarrollo de capacidades, ordenamiento territorial y medio ambiente. Es en este último eje donde se integra el ordenamiento territorial 
Arquitectura +

ISSN: 2518-2943

www.revistas.uni.edu.ni/index.php?journ

$\mathrm{al}=$ arquitectura

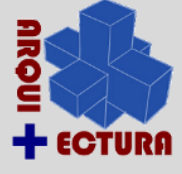

Vol. 1- Número 2 / Diciembre 2016

Gestión de riesgo con enfoque en ordenamiento territorial en el casco urbano del municipio de San Nicolás, departamento de Estelí/

Paizano, Martha Alicia/ marthapaizano@gmail.com en el plan de gestión; se propone la zonificación del territorio en función al riesgo por movimientos de laderas, basándose en el Decreto 78-2002: De Normas, Pautas y Criterios para el Ordenamiento Territorial.

\section{Palabras claves}

Amenaza, gestión de riesgos, ordenamiento territorial, vulnerabilidad, Estelí

\section{Introducción}

Nicaragua presenta condiciones climáticas y geológicas que favorecen la presencia de fenómenos naturales como: sismos, erupciones volcánicas, entre otros, que se presentan periódicamente o sin previo aviso. Modificando de forma casi imperceptible el territorio o provocando grandes desastres que afectan la calidad de vida de los habitantes. Las amenazas provenientes de fenómenos naturales no se pueden evitar porque son inherentes al ciclo natural del planeta Tierra. Sin embargo, se puede intervenir en la reducción de los niveles de vulnerabilidad que existen en el territorio, debido a factores como la inadecuada ubicación de las áreas habitacionales, técnicas y materiales de construcción inapropiados, carencia de organización para prevención de desastres, entre otros.

Uno de los principales problemas consiste en la falta de planificación en el territorio, lo cual se traduce en consecuencias adversas como el incremento de condiciones de vulnerabilidad y la degradación del medioambiente, que se convierten en un obstáculo para alcanzar el desarrollo humano en paralelo con el aprovechamiento sostenible de los recursos. Tomando en cuenta que en la región se está produciendo un acelerado incremento poblacional, que demanda mayor cantidad de recursos para consumo y terrenos para habitar, por lo que cada vez se dan mayores desequilibrios ambientales.

El casco urbano del municipio de San Nicolás (Figura 1) presenta amenazas de gran trascendencia como los movimientos de ladera y las inundaciones. Además de vulnerabilidades como la carencia de planes de gestión de riesgos y ordenamiento territorial que ha provocado el inadecuado uso del suelo; falta de recursos económicos y técnicos para la planificación, poca comunicación institucional, entre otros.

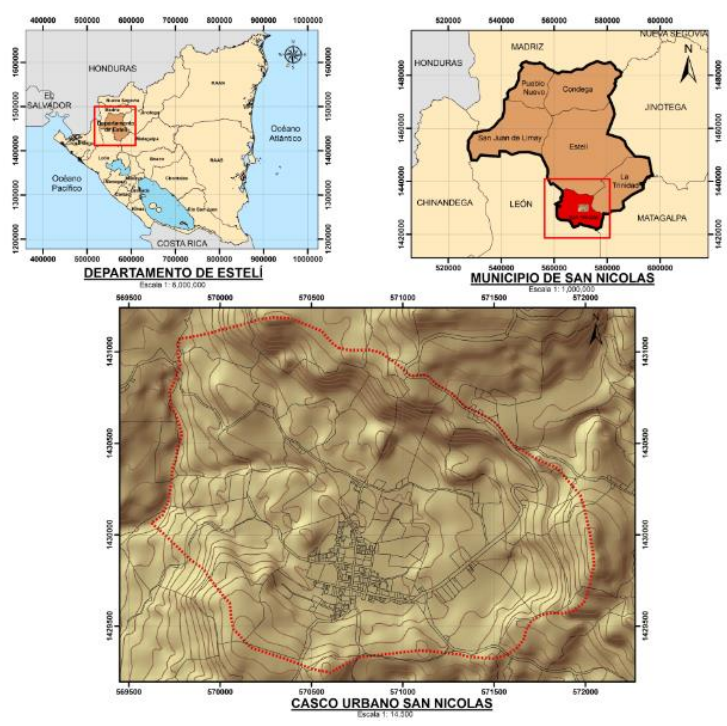

Figura 1: Mapa de localización del área de estudio.

Como una propuesta para contribuir en la reducción de las condiciones que hacen vulnerable el área de estudio, se elaboró un plan de gestión de riesgos con enfoque en ordenamiento territorial en función del riesgo ante movimientos de laderas.

\section{Metodología}

1. Recopilación de información y análisis de datos

2. Trabajo de campo

3. Procesamiento de la información: 


\section{Arquitectura + \\ ISSN: 2518-2943 \\ www.revistas.uni.edu.ni/index.php?journ \\ $\mathrm{al}=$ arquitectura}

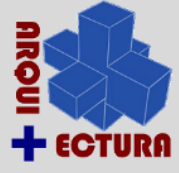

Vol. 1- Número 2 / Diciembre 2016

Gestión de riesgo con enfoque en ordenamiento territorial en el casco urbano del municipio de San Nicolás, departamento de Estelí/

Paizano, Martha Alicia/ marthapaizano@gmail.com
- Elaboración del diagnóstico.

- Identificación de las amenazas y vulnerabilidades en el territorio.

- Evaluación de la susceptibilidad a movimientos de laderas.

- Determinación de la vulnerabilidad, utilizando el método de Rosales (2001).

4. Gestión de riesgos

Específicamente la etapa de Gestión de riesgos se elaboró con el siguiente método (Figura 2): proponen 2 programas, los que a su vez están conformados por 8 proyectos.

- $\quad$ Para el eje de desarrollo social, se plantean 2 estrategias: la primera es Dotación, ampliación y mejoramiento de los servicios básicos e infraestructura; para la cual se propone 1 programa, que está conformado por 10 proyectos. La segunda estrategia es: Dotación, rehabilitación y mejoramiento del equipamiento urbano; para la cual se proponen 2 programas, que a su vez están conformados por 14 proyectos.
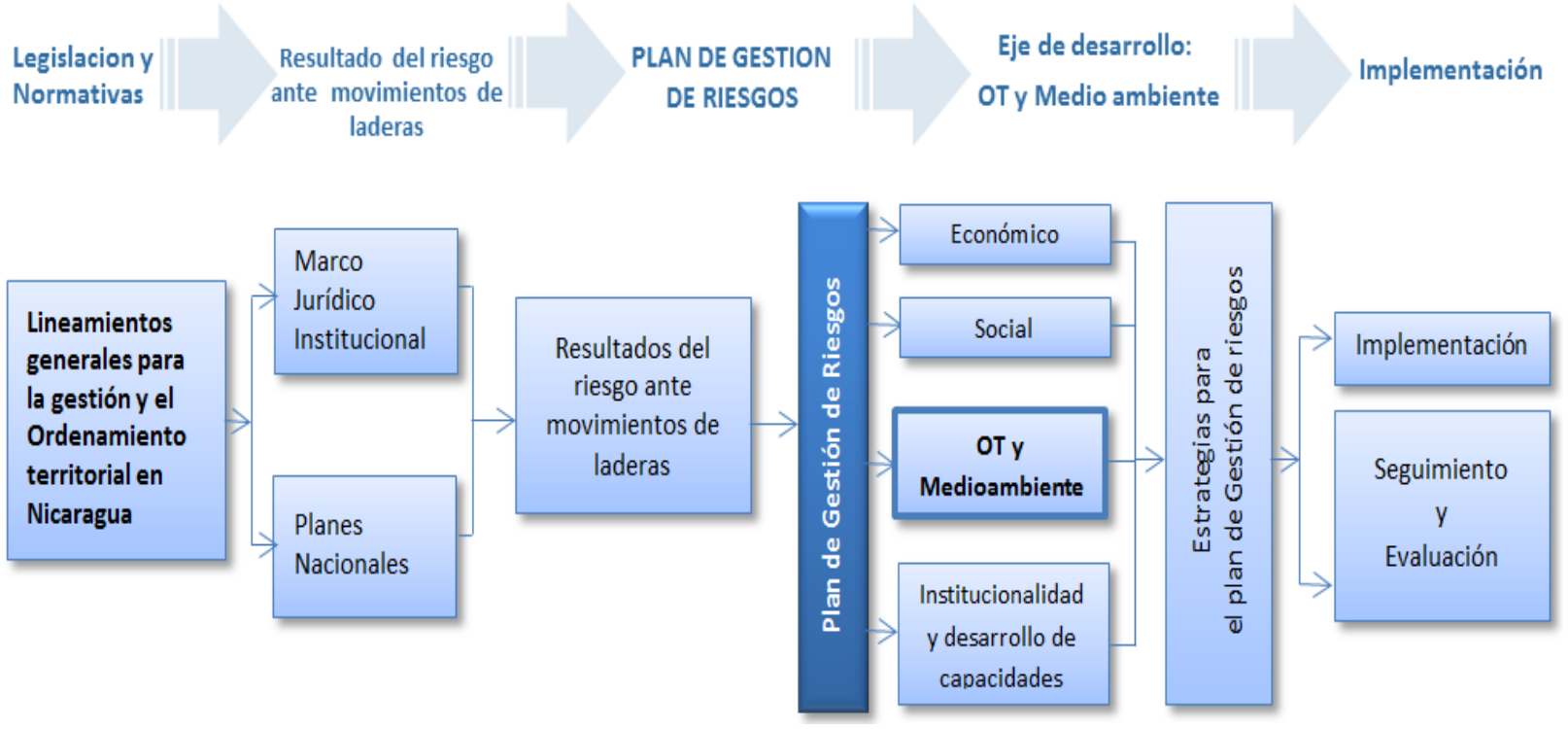

Figura 2: Método para la gestión de riesgo, modificada de Mendoza (2011) y Comisión Europea

(2008).

\section{Desarrollo y Resultados}

Las estrategias de la intervención se formularon en correspondencia a los ejes de desarrollo con enfoque a la reducción de desastres, siendo su contenido:

- Para el eje de desarrollo económico, se plantea una estrategia: Impulso de las actividades económicas en el territorio. Para lograrlo se

- Para el eje de desarrollo Ordenamiento Territorial y medio ambiente, se plantea 1 estrategia: Mejoramiento de la calidad de vida de los habitantes a partir del ordenamiento territorial, con énfasis en las zonas afectadas por Movimientos de laderas; para la cual se proponen 2 programas, que están conformados a su vez por 3 proyectos. 


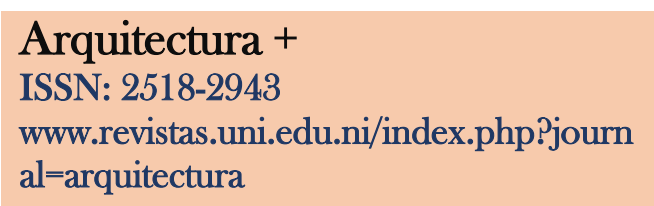

Arquitectura +

$\mathrm{al}=$ arquitectura

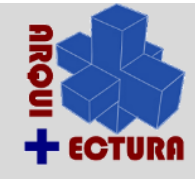

Vol. 1- Número 2 / Diciembre 2016

Gestión de riesgo con enfoque en ordenamiento territorial en el casco urbano del municipio de San Nicolás, departamento de Estelí/ Paizano, Martha Alicia/ marthapaizano@gmail.com

Para el eje de desarrollo Institucionalidad y desarrollo de capacidades, se plantea 1 estrategia: Fortalecimiento de las capacidades de los actores claves para la reducción de desastres; para la cual se proponen 4 programas, que a su vez están conformados por 26 proyectos.

Los proyectos del eje ordenamiento territorial y medio ambiente son:

1. Zonificación territorial en base al riesgo por movimientos de laderas (Figura 3).

Se han propuesto siete zonas, tomando en cuenta el contenido del Decreto 78-2002: De Normas, Pautas y Criterios para el ordenamiento territorial, las que se detallan a continuación:

- Zona de vivienda de baja densidad: Actualmente la densidad en toda la zona urbana ocupada es baja, se recomienda mantenerse en este nivel.

- Zona no apta para asentamientos humanos: corresponde al territorio que presenta un nivel de Riesgo Muy Alto ante movimientos de laderas, que además se encuentra afectado por el drenaje El Puente, que es un afluente del río Quebrada Grande. Se recomienda reubicar las viviendas que se encuentran en esta zona, o asumir el costo de obras de mitigación para reducir su vulnerabilidad.

- Zona urbana de expansión: se propone para esta zona los terrenos ubicados en la parte Noreste, debido a que el nivel de riesgo está entre Bajo y Moderado; aunque siempre existen restricciones, como que esta zona está afectada por dos ramales tributarios correspondientes al drenaje El Puente. El territorio puede ser ocupado, pero se deben cumplir estrictamente los retiros correspondientes en la Ley $\mathrm{N}^{\circ}$ 559: Ley Especial de delitos contra el Medio ambiente y los Recursos Naturales, que en su Artículo 57 Perímetro para Corte de Árboles, establece un retiro de 200 metros medidos de cada orilla de las vertientes en toda la extensión de su curso.

Aunque se propone esta zona, debido a que siempre se encuentra afectada por amenazas de inundación y encontrándose el nivel de Riesgo entre bajo y moderado; lo ideal es definir otras zonas de expansión fuera del área de estudio.

- Zona urbana de consolidación: Es la zona constituida por las áreas baldías dentro del límite de la zona urbana ocupada, que se habilitará para desarrollo urbano durante la vigencia del Plan de Ordenamiento.

- Zona para la recreación: Regularmente se consideran áreas abiertas, o áreas libres, pero abarcan los espacios de uso público o privado donde la gente asiste en diversidad de frecuencias e intensidades Comprende espacios de encuentro, parques, áreas deportivas y sitios de riquezas paisajística. En el caso del área de estudio se encuentran: el parque y el estadio municipal.

- Zona agrícola con limitaciones: se propone este uso en la parte Este de la Microcuenca, pero siempre y cuando se desarrolle la actividad agrícola con prácticas adecuadas, además de la adopción de sistemas con enfoque a la Sostenibilidad de los recursos, como la Agroecología.

- Zonas de Protección Natural, que en este caso, está constituida por:

a) Zona de protección hídrica: Aquellas áreas que se destinan para la protección de cuerpos de agua o zonas de recarga de acuíferos subterráneos. Para los recursos de agua superficial se cumplirá la norma de 200 metros establecida por el Reglamento Forestal.

b) Zona de protección de laderas: Aquellas áreas de altas pendientes cuya intervención puede 


\begin{tabular}{l}
$\begin{array}{l}\text { Arquitectura + } \\
\text { ISSN: 2518-2943 } \\
\text { www.revistas.uni.edu.ni/index.php?journ } \\
\text { al=arquitectura }\end{array}$ \\
$\begin{array}{l}\text { Vol. 1- Número 2 / Diciembre 2016 } \\
\text { Gestión de riesgo con enfoque en } \\
\text { ordenamiento territorial en el casco } \\
\text { urbano del municipio de San Nicolás, } \\
\text { departamento de Estelí/ } \\
\text { Paizano, Martha Alicia/ } \\
\text { marthapaizano@gmail.com }\end{array}$ \\
\hline
\end{tabular}

causar procesos erosivos. Además en esta zona el riesgo por Movimientos de laderas es Alto.

c) Zona de protección de biodiversidad: Aquellas áreas de protección de hábitats de especies de flora y fauna nativas, incluyendo bosques naturales primarios o secundarios, humedales $\mathrm{u}$ otras formaciones vegetales.
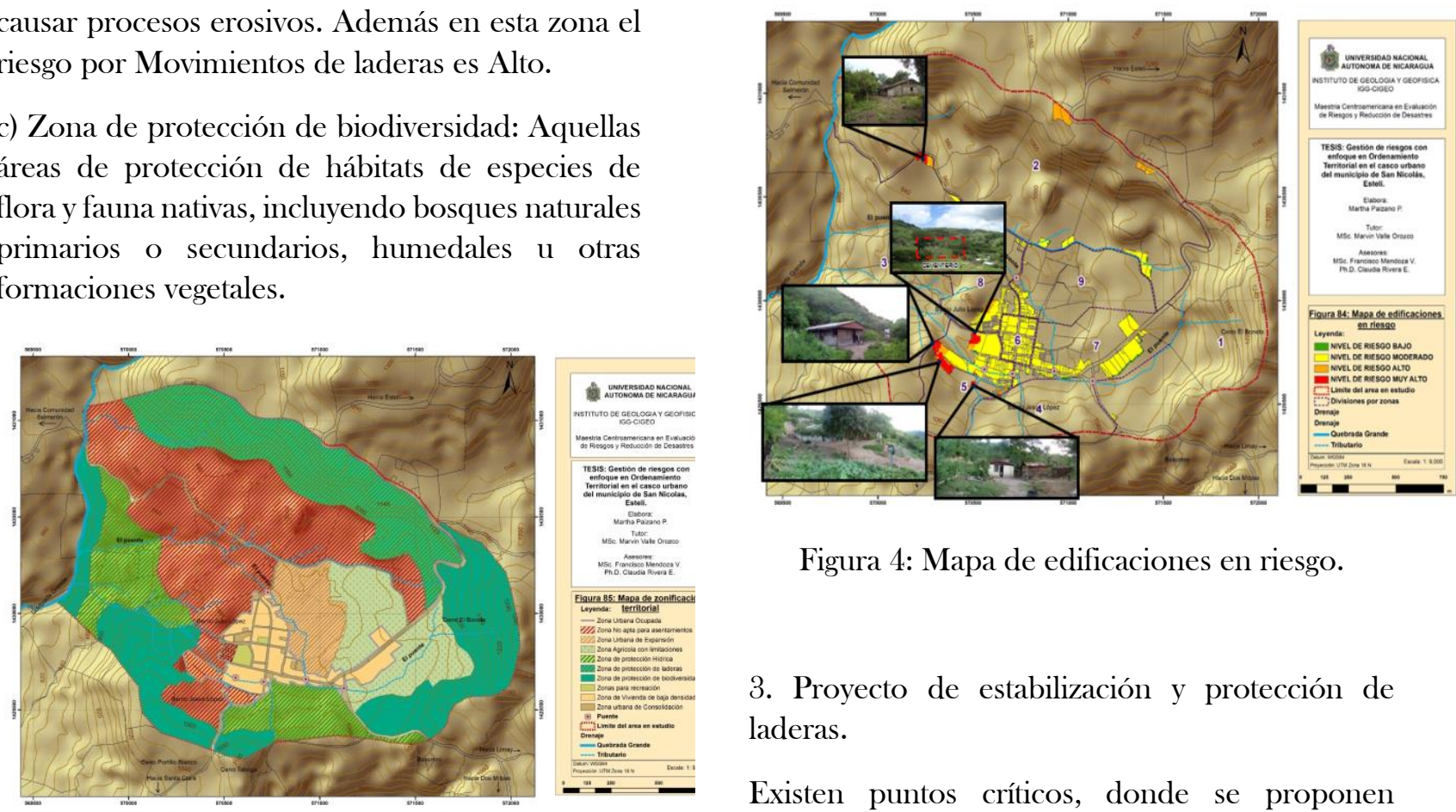

Figura 4: Mapa de edificaciones en riesgo.

Figura 3: Mapa de zonificación territorial.

2. Reubicación de las viviendas que se localizan en zonas de riesgo.

Se deben reubicar las viviendas ubicadas en las zonas donde el Riesgo tiene un Nivel Muy alto y Alto.

Se encuentran 5 edificaciones en riesgo muy Alto y 13 edificaciones en riesgo Alto, para un total de 18 edificaciones que se deben reubicar (Figura 4). Una de estas edificaciones es el Cementerio Municipal.

3. Proyecto de estabilización y protección de laderas.

Existen puntos críticos, donde se proponen alternativas de solución. Estas son algunas técnicas para estabilización y protección de laderas, pero están sujetas a estudios técnicos más detallados y locales. A continuación las propuestas:

- Viviendas a orillas de barranco en la Zona $\mathrm{N}^{\circ}$ 8: se propone la opción de diseño para un muro de suelo reforzado con geosintéticos sobre un talud con pendiente, basado en retención de tierra en Geobolsas (Figura 5), que es una solución muy versátil para resolver problemas de erosión a costos razonables. Este talud fue resuelto empleando un apilado de Geobolsas ancladas al macizo de tierra con geogrillas que se meten en el terreno y finalmente con una cara del muro vista revestida con mallas antierosivas, que favorecen el rápido desarrollo de una vegetación autóctona. 
Arquitectura +

ISSN: 2518-2943

www.revistas.uni.edu.ni/index.php?journ

$\mathrm{al}=$ arquitectura

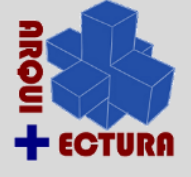

Vol. 1- Número 2 / Diciembre 2016

Gestión de riesgo con enfoque en ordenamiento territorial en el casco urbano del municipio de San Nicolás, departamento de Estelí/

Paizano, Martha Alicia/ marthapaizano@gmail.com

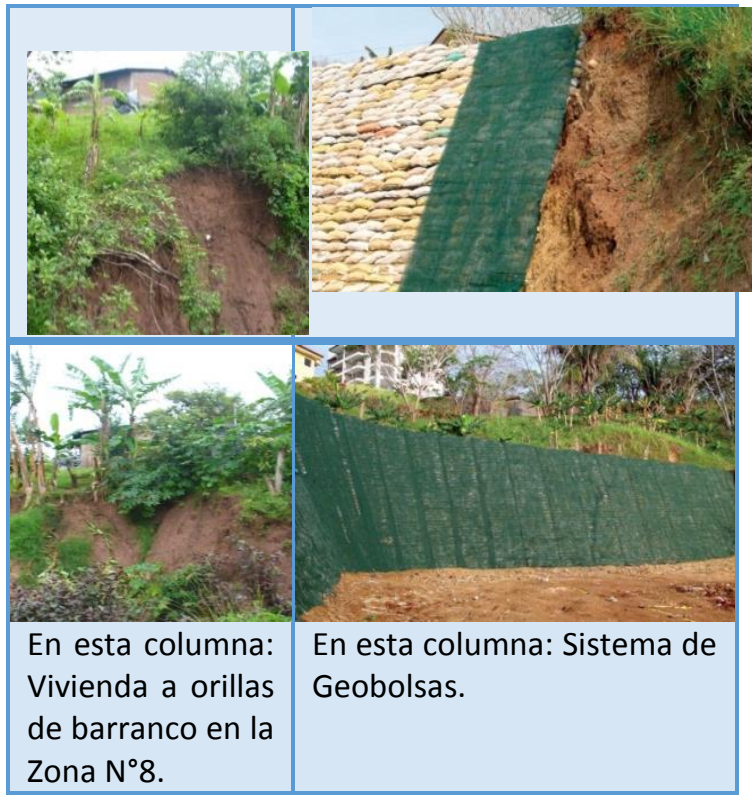

Figura 5: Sistema de Geobolsas para protección de taludes.

- Carretera principal: presenta un problema de derrumbes, es uno de los puntos críticos ya que es de vital importancia esta vía, porque es la única que comunica al municipio de San Nicolás con la cabecera departamental Estelí. Se propone la revegetación de las laderas (Figura 6) con el sistema de hidrosiembra, el cual consiste en la restauración del manto vegetal mediante la repoblación con especies herbáceas, arbustivas y arbóreas, de los sectores erosionados o dañados.

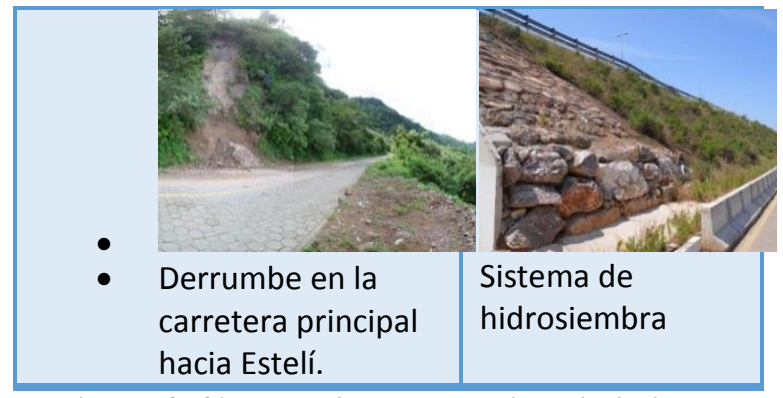

Figura 6: Sistema de revegetación de laderas
- Reforestación: Se recomienda la reforestación en las zonas afectadas por deforestación, utilizando técnicas que disminuyan la erosión del suelo, como el sistema tresbolillo o siguiendo las curvas de nivel (Figura 7). Este sistema consiste en establecer las plantaciones distribuyendo las plantas a distanciamientos iguales formando triángulos.

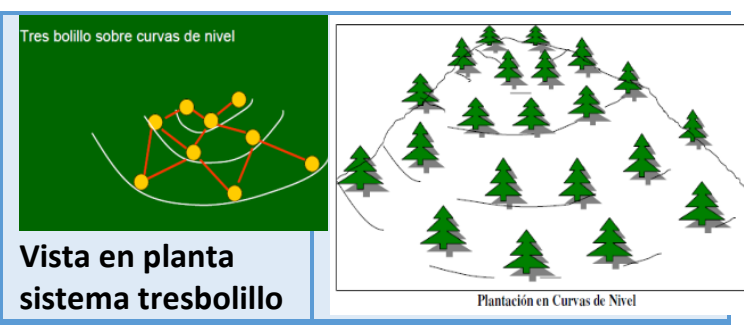

Figura 7: Sistema tresbolillo o siguiendo las curvas de nivel.

\section{Conclusiones}

1. El diagnóstico realizado revela que algunas de las principales problemáticas están relacionadas con la economía local, cobertura de los servicios básicos, estado físico y carencia de equipamiento e infraestructura, inadecuadas prácticas agrícolas en la microcuenca, exposición de edificaciones al riesgo por movimientos de laderas e inundaciones.

2. En el área de estudio se manifiestan amenazas: sísmicas, deslizamientos, inundaciones, sequias, vientos fuertes, deforestación, incendios forestales y contaminación ambiental.

3. La vulnerabilidad es moderada en las zonas 1, 2, 6, 7, 8 y 9. Las zonas 3 y 5 presentan vulnerabilidad alta. La zona 4 permanece dentro de la vulnerabilidad baja debido a que no existe medio construido, es decir, no hay habitantes en esta zona. 


Arquitectura +
ISSN: $2518-2943$
www.revistas.uni.edu.ni/index.php?journ
al=arquitectura

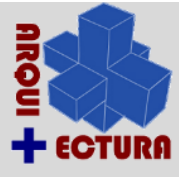

Vol. 1- Número 2 / Diciembre 2016

Gestión de riesgo con enfoque en ordenamiento territorial en el casco urbano del municipio de San Nicolás, departamento de Estelí/

Paizano, Martha Alicia/ marthapaizano@gmail.com
4. De acuerdo a los resultados de la evaluación del riesgo ante movimientos de laderas, el $44 \%$ del territorio presenta riesgo moderado, seguido por el riesgo alto con un $31 \%$; riesgo bajo con $22 \%$ y en menor porcentaje se expresa el riesgo muy alto con $3 \%$.

5. De las edificaciones existentes en el área de estudio, 4 viviendas y el cementerio presentan riesgo muy alto y otras 13 viviendas se encuentran en riesgo alto; por lo que son propensos a sufrir daños por encontrarse sobre áreas en las que se pueden dar movimientos de laderas.

6. Para contribuir a la disminución de la problemática existente, se elaboró el Plan de gestión de riesgos, donde se han propuesto cuatro ejes de desarrollo: Económico; Social; Ordenamiento territorial y Medio ambiente; e Institucionalidad y desarrollo de capacidades.

7. El eje de desarrollo Ordenamiento territorial y Medio ambiente, está conformado por dos programas y tres proyectos. Los proyectos propuestos son zonificación territorial en base al riesgo por movimientos de laderas, reubicación de edificaciones que se localizan en zonas de riesgos, y estabilización y protección de laderas.

8. La zonificación propuesta contribuye a la reducción de los riesgos ante movimientos de laderas mediante la definición de: zona no apta para asentamientos humanos, zona urbana de expansión, zonas de protección natural, zona de vivienda de baja densidad, zona urbana de consolidación y zona agrícola con limitaciones.

\section{Recomendaciones}

1. La Zonificación territorial en base al riesgo por movimientos de laderas, representa un punto de partida hacia la adecuada planificación en el territorio, por lo que se debe incorporar las medidas propuestas en una planificación más general, que comprenda un Plan General de Desarrollo
Urbano y un Plan Regulador para el municipio de San Nicolás.

2. En el casco urbano actual de San Nicolás se debe mantener la densidad baja de edificaciones y control por parte de la municipalidad para que no se continúe dando el asentamiento en zonas de riesgo.

3. Aunque se ha propuesto una zona urbana de expansión, en ésta existen restricciones; por lo cual es más adecuado definir otras zonas de crecimiento, consolidación y desarrollo fuera de la microcuenca.

4. Se recomienda la reubicación a corto plazo de las viviendas que se encuentran en zonas de riesgo muy alto y alto, o asumir el costo de realizar obras de mitigación. En este sentido, un proyecto habitacional para la reubicación de estas edificaciones puede ser financiado con fondos del gobierno local o por cooperación externa.

5. La zona definida como no apta para asentamientos humanos, también puede incorporarse al Plan como una zona de recuperación, siempre y cuando sea tratada para su rehabilitación y posteriormente utilizarse como zona de protección natural.

6. Debido a su trascendencia y fuerte influencia sobre el área de estudio, se recomienda realizar un estudio sobre el riesgo ante inundaciones y proponer obras de mitigación que complementen las que ya han sido propuestas para el riesgo ante Movimientos de laderas.

7. Es importante fomentar el cumplimiento del Reglamento Nacional de la Construcción en el proceso de diseño y construcción de nuevas edificaciones, mano de obra calificada y técnicas constructivas apropiadas.

\section{Bibliografías y Referencias}

1. Comisión Europea/Predecan/Comunidad Andina. (2008). Plan de Gestión Local del Riesgo de Desastres Calca, Perú. Noviembre, 2008. Recuperado el 29 de octubre de 2011, de 
Arquitectura +

ISSN: 2518-2943

www.revistas.uni.edu.ni/index.php?journ

$\mathrm{al}=$ arquitectura

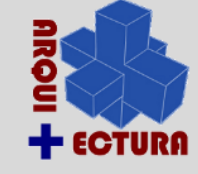

Vol. 1- Número 2 / Diciembre 2016

Gestión de riesgo con enfoque en ordenamiento territorial en el casco urbano del municipio de San Nicolás, departamento de Estelí/

Paizano, Martha Alicia/ marthapaizano@gmail.com http://www.comunidadandina.org/predecan/ doc/libros/pp/pe/Plan+Gestion+Local +Riesg $\mathrm{o}+$ Calcal.pdf

2. González (2011). Evaluación del riesgo ante movimientos de laderas en el casco urbano del municipio de San Nicolás, departamento de Estelí año 2011. Tesis de Maestría en proceso, Centro de Investigaciones Geocientíficas, Universidad Nacional Autónoma de Nicaragua. Managua, 2011.

3. Mendoza (2011). "Entrevista a experto en el tema de Gestión de Riesgos”. En Universidad Nacional de Ingeniería. Director Programa de Estudios Ambientales Urbanos y Territoriales (PEAUT). Managua, Nicaragua, 2011.

4. Rosales (2001). Metodología para evaluar la Vulnerabilidad en Asentamientos Humanos. Managua, Nicaragua.

5. Instrumentos de apoyo para el Análisis y la Gestión de Riesgos Naturales en el ámbito municipal de Nicaragua - Guía del especialista. (2002). COSUDE \& ALARN, PREVAC (Programa de Prevención y Preparación ante Desastres).

6. REBOTIER, JULIEN. (2014). La vulnerabilidad urbana: entre reducción de riesgo y emancipación social. Ejemplos en Venezuela. Pueblos indígenas y descolonización \#38 Polis [En línea], 38 | 2014, Publicado el 10 septiembre 2014.

7. La Ley 337- Ley Creadora Del Sistema Nacional para la Prevención Mitigación y Atención de Desastres. Marco Legal República de Nicaragua.

8. Normas Mínimas de Dimensionamiento para Desarrollos Habitacionales - NTON 11 013-04.

9. Decreto 782002 - Normas, pautas y criterios para el ordenamiento territorial. Marco Legal República de Nicaragua.

\section{Anexos}

1. Matriz de programas, proyectos y actores.

\section{EJE DE DESARROLLO: ECONÓMICO}

\section{Estrategia 1: Impulso de las actividades económicas en el territorio}

\section{Programa 1: Actividades económicas}

\section{Proyectos:}

1. Impulsar la creación de Asociaciones de productores, para facilitar las gestiones de financiamiento.

2. Proyecto de Ecoturismo comunitario para aprovechar de manera sostenible los recursos naturales existentes en el territorio.

3. Construcción de un Mercado municipal, para impulsar el comercio local.

4. Apertura de Centros de estudios técnicos orientados al desarrollo agroforestal en el municipio.

5. Proyecto de impulso de la Agroecología, como los sistemas agroforestales y prácticas agronómicas de conservación de los suelos.

6. Creación de contratos de transferencia de riesgo a nivel individual con compañías de seguros o reaseguros, como mecanismos para que las familias y los productores puedan solventar los efectos adversos en caso de desastres. 
Arquitectura +

ISSN: 2518-2943

www.revistas.uni.edu.ni/index.php?journ

al=arquitectura

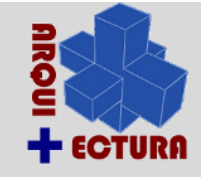

Vol. 1- Número 2 / Diciembre 2016

Gestión de riesgo con enfoque en ordenamiento territorial en el casco urbano del municipio de San Nicolás, departamento de Estelí/

Paizano, Martha Alicia/ marthapaizano@gmail.com

\section{Programa 2: Capacitación \\ Proyectos:}

1. Jornadas de capacitación para los pobladores que están involucrados en el proyecto de ecoturismo comunitario.

2. Asesoría técnica acerca de Agroecología.

Actores: Alcaldía municipal, ONG afines, MTI, MARENA, Organización comunal

\section{EJE DE DESARROLLO: SOCIAL ${ }^{1}$}

Estrategia 1: Dotación, ampliación y mejoramiento de los servicios básicos e infraestructura

\section{Programa 1: Servicios básicos e infraestructura \\ Proyectos:}

1. Instalación de sistema de drenaje sanitario.

2. Servicio de recolección de basura y adecuado tratamiento de los desechos.

3. Ampliación del sistema de alumbrado público.

4. Mejoramiento y ampliación del servicio de agua potable.

5. Mejoramiento y ampliación del servicio de energía eléctrica.

6. Mejoramiento del tramo de carretera afectado por derrumbes en el acceso al casco urbano.

7. Adoquinado de camino hacia las comunidades del área rural, con el objetivo de impulsar el desarrollo de la producción agrícola.

8. Construcción de un puente en el camino hacia Salmerón, en el punto donde atraviesa el rio Quebrada Grande.

9. Mejoramiento de dos puentes en la zona $\mathrm{N}^{\circ} 7$ y un puente en la zona $\mathrm{N}^{\circ} 5$.

10. Construcción de andenes peatonales y cunetas en las calles que no cuentan con éstos.

\section{Estrategia 2: Dotación, rehabilitación y mejoramiento del equipamiento urbano}

\section{Programa 1: Equipamiento urbano \\ Proyectos:}

1. Construcción de albergues seguros.

2. Señalización de las rutas de evacuación.

3. Construcción de Casa comunal y cultural.

4. Construcción de Centro de Desarrollo Infantil y comedor infantil.

5. Rehabilitación de parque municipal.

6. Mejoramiento y mantenimiento al estadio municipal.

7. Mejoramiento del actual cementerio.

8. Construcción de nuevo cementerio en sitio seguro.

9. Mejoramiento de viviendas que presentan mal estado físico.

10.Mejoramiento de las instalaciones del centro de salud, Preescolar y Biblioteca municipal.

\footnotetext{
${ }^{1}$ Se realizan las propuestas en base a las categorías poblacionales para fines de equipamiento e infraestructura, en este caso le corresponde como Villa de acuerdo a la proyección poblacional (Ver Anexos 3).
} 
Arquitectura +

ISSN: 2518-2943

www.revistas.uni.edu.ni/index.php?journ

al=arquitectura

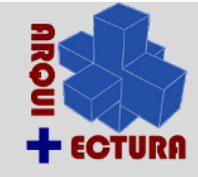

Vol. 1- Número 2 / Diciembre 2016

Gestión de riesgo con enfoque en ordenamiento territorial en el casco urbano del municipio de San Nicolás, departamento de Estelí/ Paizano, Martha Alicia/ marthapaizano@gmail.com

11. Construcción de viviendas seguras para reducir la vulnerabilidad estructural ante desastres, en el caso de aquellas que se deben reubicar.

\section{Programa 2: Medidas no estructurales \\ Proyectos:}

1. Concientización hacia la población acerca de la preservación del equipamiento urbano.

2. Concientización acerca de educación ambiental, con el objetivo de evitar la eliminación de residuos sólidos en espacios públicos, calles, quebradas, entre otros.

3. Reforzamiento de las funciones de control urbano para impedir la eliminación de residuos sólidos y de líquidos residuales en espacios públicos, ríos y quebradas. Se pueden emitir multas para estimular el cumplimiento de estas medidas.

Actores: Alcaldía municipal, ONG afines, Organización comunal.

\section{EJE DE DESARROLLO: ORDENAMIENTO TERRITORIAL Y MEDIO AMBIENTE}

Estrategia 1: Mejoramiento de la calidad de vida de los habitantes a partir del ordenamiento territorial, con énfasis en las zonas afectadas por Movimientos de laderas Programa 1: Gestión prospectiva (prevención)

\section{Proyectos:}

1. Zonificación territorial en base al riesgo por movimientos de laderas.

\section{Programa 2: Gestión Correctiva (Mitigación) Proyectos:}

1. Reubicación de las viviendas que se localizan en zonas de riesgo.

2. Proyectos de estabilización y protección de laderas.

Actores: Alcaldía municipal, Organización comunal, MARENA, MAGFOR, MTI, ONG afines.

EJE DE DESARROLLO: INSTITUCIONALIDAD Y DESARROLLO DE CAPACIDADES

Estrategia 1: Fortalecimiento de las capacidades de los actores claves para la reducción de desastres.

\section{Programa 1: Medidas no estructurales para prevención de desastres}

\section{Proyectos:}

1. Monitoreo de amenazas e instalación de sistemas de alerta temprana.

2. Fortalecimiento institucional en gestión de riesgos.

3. Mejoramiento de la coordinación entre todos los actores claves para la gestión de los riesgos a nivel local.

4. Reactivación de los Comités Municipales de Prevención de Desastres (COMUPRED).

5. Mejoramiento de la coordinación con los demás niveles administrativos en la región y a nivel municipal, sobre todo con aquellos involucrados en la gestión de riesgos: SINAPRED, Defensa Civil, entre otros.

6. Fortalecimiento y promoción de la participación comunitaria en las acciones que conllevan los procesos de gestión del riesgo en el territorio.

7. Campañas educativas de preservación medioambiental.

8. Cumplimiento del Reglamento nacional de la construcción en el proceso de diseño y construcción de nuevas edificaciones, además de disponer de mano de obra calificada y técnicas constructivas apropiadas con el entorno. 


\author{
Arquitectura + \\ ISSN: 2518-2943 \\ www.revistas.uni.edu.ni/index.php?journ \\ $\mathrm{al}=$ arquitectura
}

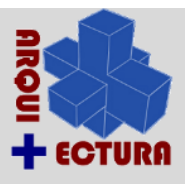

Vol. 1- Número 2 / Diciembre 2016

Gestión de riesgo con enfoque en ordenamiento territorial en el casco urbano del municipio de San Nicolás, departamento de Estelí/ Paizano, Martha Alicia/ marthapaizano@gmail.com

9. Divulgación de los planes de prevención de riesgos, a través del impulso de jornadas de capacitación, para informar a la comunidad acerca de la ubicación de los albergues y sitios seguros, así como cuáles son las rutas de evacuación.

10.Asesoría sobre técnicas de autoconstrucción popular y mejoramiento de edificaciones.

11.Promoción y fortalecimiento de programas que desarrolla el Ministerio de educación (MINED) en que se deben impartir conocimientos acerca de la gestión del riesgo y valores de respeto y preservación de los recursos medioambientales.

12. Actualización del Plan de gestión de riesgos, con el objetivo de contar con información reciente acerca de los procesos cambiantes que experimenta el territorio, los cuales pueden ser: geológicos, geomorfológicos, climáticos, entre otros.

\section{Programa 2: Protección Financiera \\ Proyectos:}

1. Fortalecimiento de fondos especializados de reservas monetarias para enfrentar desastres a nivel nacional.

2. Creación de contratos de transferencia de riesgo con compañías de seguros o reaseguros, como mecanismos para enfrentar adecuadamente las labores de respuesta, rehabilitación y reconstrucción.

\section{Programa 3: Preparación para la respuesta \\ Proyectos:}

1. Actualización del Plan de respuesta municipal con enfoque en la Gestión del riesgo.

2. Dotación de mayor número de equipos para la respuesta (vehículos, radio-comunicadores, teléfonos, entre otros).

3. Fortalecimiento de la Organización para el desastre, asegurando que cada una de entidades (COMUPRED, CODE y demás comisiones) cumpla con su responsabilidad en la ejecución de las fases de respuesta.

4. Fortalecimiento para la Organización de la evacuación, para asegurar que exista coordinación entre las entidades involucradas, conocimiento de su función y de los medios disponibles.

5. Creación de sistema de vigilancia epidemiológica en caso de desastres.

\section{Programa 4: Rehabilitación y Reconstrucción}

\section{Proyectos:}

1. Elaboración de Evaluación de Daños y Análisis de Necesidades (EDAN).

2. Creación de viviendas y albergues temporales, que cuenten con las condiciones óptimas de espacio, líneas vitales y atención en salud.

3. Rehabilitación de servicios básicos, infraestructura y equipamiento que resultan parcialmente dañados después del desastre.

4. Reconstrucción de servicios básicos, infraestructura y equipamiento que resultan destruidos después del desastre.

5. Reactivación económica y la generación de empleo en el nivel local.

6. Reubicación de viviendas afectadas y de las asentadas sobre sitios de riesgo.

7. Construcción de nuevas viviendas en sitios seguros.

Actores: Alcaldía municipal, MINED, MINSA, SINAPRED, MARENA, MAGFOR, MTI, Policía Nacional, Organización comunal, Población local, Asamblea Nacional, Gobierno Central, ONG afines, Cooperación Internacional, entre otros. 